\title{
Quasar absorption lines at high redshift: through a glass darkly
}

\author{
Steven R. Furlanetto \\ Department of Physics, Mathematics, and Astronomy; California Institute of Technology; \\ Mail Code 130-33; Pasadena, CA 91125, USA \\ email: sfurlane@tapir.caltech.edu
}

\begin{abstract}
Thanks to the Sloan Digital Sky Survey, we now know that the Universe becomes opaque to $\operatorname{Ly} \alpha$ photons at $z \sim 6$. This presents an obvious challenge for attempts to study the Universe at high redshifts with quasar absorption lines. I discuss two ways to extract more information about reionisation than the simple presence of complete Gunn-Peterson absorption. First, we still expect to see metal line absorption red-ward of Ly $\alpha$. This will allow us to probe the early phases of metal enrichment in the intergalactic medium; the lines can be quite strong because the enriched regions are relatively compact. This is particularly important because metals are a crucial feedback mechanism in the early Universe. Second, we expect to see isolated $\operatorname{Ly} \alpha$ transmission features even before the final stages of reionisation. These features allow us to learn about the topology of reionisation and the condensation of the cosmic web at $z \sim 6$.
\end{abstract}

\section{Introduction}

The last several years have seen a great leap forward in our understanding of the highredshift Universe and, in particular, the reionisation of hydrogen at $z>6$. The discovery of several bright quasars at this epoch through the Sloan Digital Sky Survey (SDSS; see Fan et al. 2001) has opened a new window for studying the high-redshift intergalactic medium (IGM). All of the quasars with $z>6.2$ show complete - or at least nearly complete - Ly $\alpha$ absorption. The appearance of this long-sought Gunn \& Peterson (1965) absorption may herald the end of the reionisation era. On the other hand, the Wilkinson Microwave Anisotropy Probe (WMAP) has recently measured a large optical depth to electron scattering for $\mathrm{CMB}$ photons, implying that reionisation began at $z \gg 6$ (Kogut et al. 2003).

Taken together, these two sets of observations imply a complex reionisation history. If galaxy properties remained constant as they grew, reionisation would proceed rapidly because structure formation is exponential at early times (Barkana \& Loeb 2001, and references therein). Reconciling the SDSS spectra and the WMAP measurements therefore requires galaxy properties to evolve significantly, most likely through some form of feedback (e.g. Cen 2003; Wyithe \& Loeb 2003). Possible feedback mechanisms include photoheating, $\mathrm{H}_{2}$ cooling, and metal enrichment. For example, recent numerical simulations suggest that metal-free stars may be very massive (Abel et al. 2002; Bromm et al. 2002) and hence efficient ionisers. However, they also produce heavy elements and presumably spread them throughout the IGM (e.g. Madau et al. 2001; Scannapieco et al. 2002), making subsequent generations of stars more normal. This scenario may also help explain the tension between the ubiquity of metals in the low- $z$ IGM and the apparent lack of dynamical effects of large-scale winds (see, e.g. A. Aguirre in this volume). Any of these feedback mechanisms can extend reionisation significantly (though they actually act too smoothly to induce "double reionisation", Furlanetto \& Loeb 2005). 
It is therefore crucial to understand how much more we can learn from quasar absorption spectra. At first sight, the prospects do not appear good: the optical depth of the fully neutral IGM is $\tau_{\alpha} \approx 6 \times 10^{5}[(1+z) / 10]^{3 / 2}$, so one might expect that quasars can only probe the tail end of reionisation. However, recent simulations and analytic models of reionisation show that "overlap" is likely to be extremely inhomogeneous (Ciardi et al. 2003; Sokasian et al. 2003, 2004; Furlanetto et al. 2004a, hereafter FZH04); in that case, reionisation is characterised by large-scale fluctuations in the ionising background. In $\S 2$ and $\S 3$, we will examine how existing data displays such inhomogeneity and consider its implications for future studies.

Another promising approach is to search for metal lines even when the Ly $\alpha$ forest is saturated, which remains possible for transitions red-ward of Ly $\alpha$. This would allow us to observe directly the earliest stages of IGM enrichment. We will examine the prospects for studying this process in $\S 4$. We will also describe how these metal transitions may yield constraints on reionisation itself.

\section{Interpreting the SDSS quasar spectra}

How much have we already learned from the existing sample of SDSS quasars? We will focus on SDSS J1030+0524 $(z=6.28)$ and SDSS J1148+5251 $(z=6.42)$, which have the highest quality spectra to date (White et al. 2003). The interpretation of J1030 seems relatively simple: it shows completely saturated absorption in all of the Lyman troughs from $z=6.19-5.96$, with an average optical depth in Ly $\alpha$ of $\tau_{\alpha}>9.9$ (at the $2 \sigma$ level). While this clearly requires a rapid increase in $x_{\mathrm{HI}}$ along this line of sight, the quantitative implications for the $x_{\mathrm{HI}}$ neutral fraction are highly uncertain. The limit averages over $\sim 100$ co-moving Mpc; actually the transmission fluctuates rapidly along with the density. In fact, transmission occurs only in rare voids (see Fig. 1). Proper interpretation of this trough therefore requires knowledge of the IGM density field. The Ly $\alpha$ trough itself places only a weak lower limit to $x_{\mathrm{HI}}$; better constraints (such as the limit quoted above) come from the weaker Ly $\beta$ and Ly $\gamma$ transitions, because they sample densities closer to the mean. Rigorously, all we can say is that $x_{\mathrm{HI}}$ increases rapidly within voids on this line of sight.

J1148 is even more difficult to interpret, as it has both an extremely faint continuum and a number of "transmission spikes" inside the troughs (several in Ly $\beta$ and one in $\operatorname{Ly} \alpha$ ). Initially, both the continuum and spikes were attributed to contamination by a $z=5$ galaxy along the line of sight. Recently, however, White et al. (2005) showed that the transmission spikes are point sources at HST resolution, implying that they must be genuine quasar light because any intervening galaxy would have been resolved. At the same time, Oh \& Furlanetto (2005) argued that the continuum was also transmitted quasar flux because it does not show the Lyman-break characteristic of a $z=5$ galaxy. They measured an average $\tau_{\gamma}=2.4 \pm 0.4$ at $z \approx 6.2$ along this line of sight; this sets a (model-dependent) limit $\tau_{\alpha}<11$ even in regions uncontaminated by obvious transmission spikes, although it is still impossible to draw firm conclusions about $x_{\mathrm{HI}}$. Thus, the two known lines of sight have dramatically different IGM properties. Both have a rapidly increasing $\tau_{\alpha}$ at $z>6$, but one remains extremely highly ionised and the other appears much more neutral. Moreover, Mesinger \& Haiman (2004) and Wyithe \& Loeb (2004) argued that $x_{\mathrm{HI}}>0.1$ by considering the proximity zone of the quasars. Even with the limited amount of data available today, we see substantial cosmic variance in reionisation on extremely large $\left(\sim 100\right.$ co-moving Mpc) scales as well as the difficulty of inferring $x_{\mathrm{HI}}$ from the observations. 


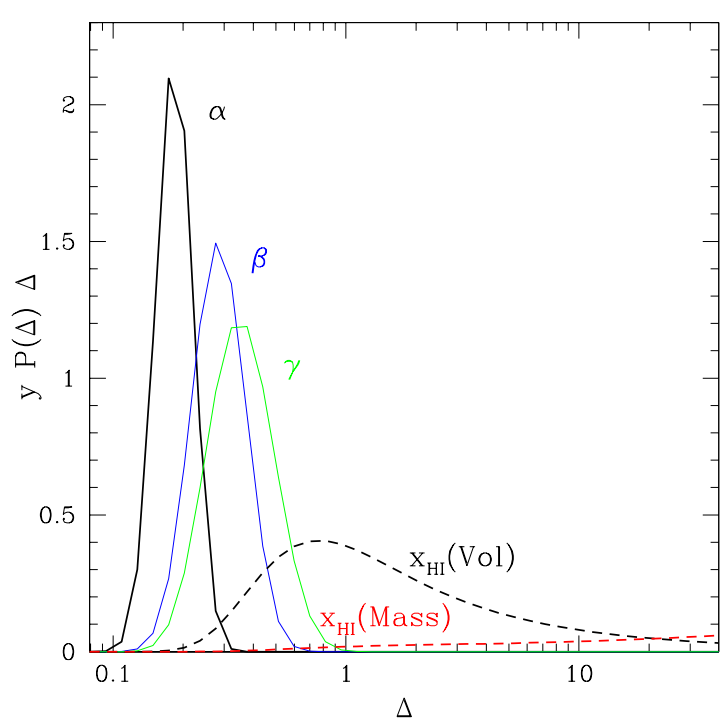

Figure 1. Fraction of transmission coming from each density $\Delta=\rho / \bar{\rho}$ in the IGM. From left to right, the solid curves show the fraction for the $\operatorname{Ly} \alpha, \operatorname{Ly} \beta$, and $\operatorname{Ly} \gamma$ transitions. The dashed curves show the fraction of the neutral gas at each density, weighted by mass and volume. We assume a uniform ionising background, isothermal gas, and the density model of Miralda-Escudé et al. (2000). From Oh \& Furlanetto (2005).

\section{Transmission gaps in the Gunn-Peterson trough}

What does this cosmic variance imply for reionisation? And how can we interpret the transmission spikes: can they even exist when $x_{\mathrm{HI}}<0.99$ ? To answer this question, note that transmission requires both a tiny local hydrogen density (either because it lies in a void or because the local ionising background is large) and a substantial distance from any other neutral patch. Because the Gunn-Peterson optical depth is so enormous, $\tau_{\alpha}$ is large even in the damping wings of the line. Barkana (2002) showed that if the HII regions around each galaxy are considered in isolation, the latter condition is impossible to fulfil unless the global neutral fraction is extremely close to zero.

However, recent numerical simulations (Ciardi et al. 2003; Sokasian et al. 2003, 2004) of reionisation have shown that such a picture does not describe the real Universe. Galaxies are rare at high redshifts and cluster strongly; they cannot be treated in isolation, because most have neighbours within their local HII regions. Thus, ionised bubbles quickly "overlap" around clusters of sources, even if most of the IGM remains neutral (Barkana \& Loeb 2004). Recently, FZH04 constructed a model for the topology of reionisation that includes these clustering effects. It begins with the simple assumption that each galaxy is able to ionise a fixed multiple of its own mass; then, using the statistics of Gaussian density fields, it predicts the distribution of ionised bubble sizes throughout reionisation. Clustering is remarkably important: while the HII regions first grow around individual galaxies, they reach typical radii $\sim 5$ co-moving Mpc when $x_{\mathrm{HI}} \approx 0.5$ and several tens of Mpc when $x_{\mathrm{HI}} \sim 0.8-0.9$. If the Universe is nearly reionised, even a small large-scale physical over-density increases the galaxy density sufficiently to ionise the entire region.

This has important implications for quasar absorption spectra (and indeed for any observable of reionisation). First, it offers a clear and intuitive way to understand the large-scale line of sight variations described in $\S 2$. Second, it suggests that transmission 


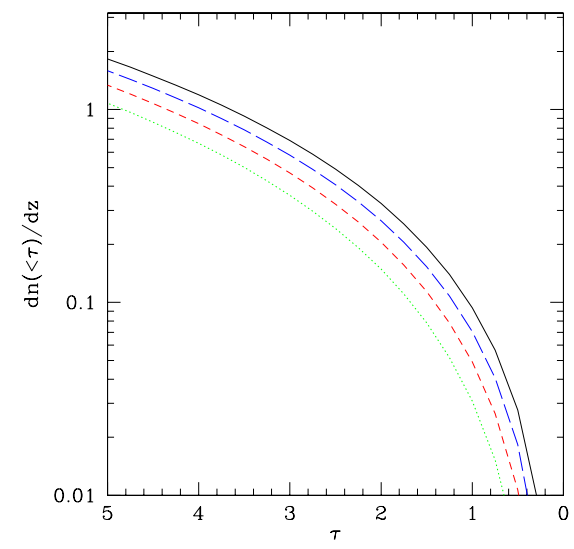

Figure 2. Expected frequency of transmission gaps (i.e. contiguous regions with central Ly $\alpha$ optical depth smaller than the specified value) at $z=6.1$ if $x_{\mathrm{HI}}=0.1,0.15,0.2$, and 0.25 (from top to bottom). From Furlanetto et al. (2004b).

gaps can appear even during reionisation. Because the ionised regions are so enormous, large swaths of the Universe are far from the nearest fully neutral gas, rendering unimportant the damping wing absorption that is so ruinous within small bubbles. Furlanetto et al. (2004b) computed the resulting transmission statistics for a simple model of the IGM density field (required to predict the distribution of $\tau_{\alpha}$, Miralda-Escudé et al. 2000). Fig. 2 illustrates their results. It shows the number of gaps per unit redshift as a function of $\tau_{\alpha}$ and $x_{\mathrm{HI}}$. Although it is by no means common, we see that transmission should not be vanishingly rare even when $x_{\mathrm{HI}} \sim 0.25$. This occurs because HII regions with sizes $>10$ co-moving Mpc (the scale at which damping wing absorption becomes small) begin to appear at this time. Any line of sight passing through such a region can show transmission. These models therefore suggest that we should not expect to see unbroken Gunn-Peterson troughs during reionisation. Instead, we expect some amount of transmission to persist until the middle stages, modulated by weak underlying large-scale density fluctuations. Thus, quasars by themselves can probe deep into the reionisation epoch.

Of course, the bubbles cannot grow infinitely large: photons must be able to propagate through the highly (though not completely) ionised cosmic web inside. They will continue to grow until their size approaches the mean free path of an ionising photon. Shortly after reionisation, at $z \sim 5.5$, the mean free path is $\sim 20$ co-moving Mpc (Fan et al. 2002); Furlanetto \& Oh (2005) showed that, according to the most popular IGM density models, this is also roughly equal to the maximum size during reionisation. However, in principle reionisation could itself affect the IGM density (by e.g. photo-evaporating minihalos, Shapiro et al. 2004), so studying the transition from a "bubble-dominated" to a "web-dominated" topology will elucidate details of substructure in the IGM. Because the maximum bubble size affects the local ionising background and hence the transmission, measuring the decrease of transmission throughout reionisation will constrain how the cosmic web comes to regulate the meta-galactic radiation field.

\section{Metal absorption lines before reionisation}

Of course, the Ly $\alpha$ transition is not the only way to study the IGM. Metal transitions red-ward of Ly $\alpha$ offer the obvious advantage of avoiding saturation. But, their absorption is not necessarily negligible: the equivalent Gunn-Peterson optical depth at $z=6$ for a 


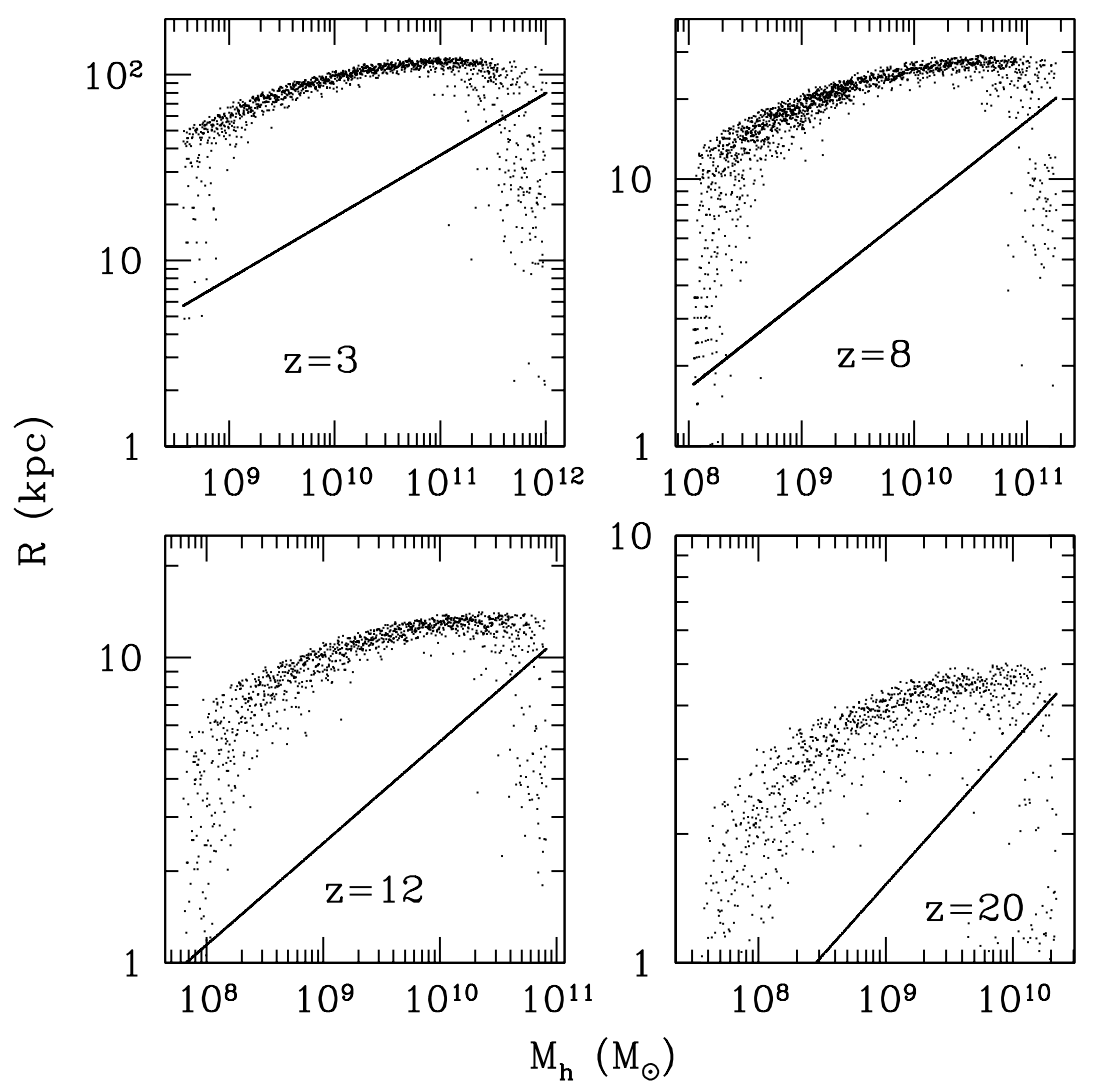

Figure 3. Physical radius of wind bubbles at various redshifts. The points are Monte Carlo realisations of a simple wind expansion model. The solid lines show the halo virial radii; note that small galaxies are much more efficient polluters than massive galaxies. From Furlanetto \& Loeb (2003).

metal transition is typically $\tau \sim 0.1$ for a metallicity $Z=10^{-2.5} \mathrm{Z}_{\odot}$ (see Table 1 of $\mathrm{Oh}$ 2002). Here we will consider what we can learn through these transitions.

\subsection{The enrichment of the IGM}

Obviously, such lines will allow us to probe the dispersal of metals throughout the early Universe. The most likely agent for enriching the IGM is supernova winds from starburst galaxies. These may be especially effective at high redshifts because of the small gravitational binding energies of the host halos (Dekel \& Silk 1986; Madau et al. 2000; Scannapieco et al. 2002). Unfortunately, we have little theoretical or observational understanding of winds, even at low and moderate redshifts. Thus predictions for metal line absorption at $z>6$ are uncertain at best; even the identity of the most relevant transitions is unclear. If the enriched medium is highly ionised, states such as CIV and SiIV are likely targets. However, earlier in reionisation metals may be confined to compact, dense shells surrounding wind hosts, or they may be distributed through a mostly neutral Universe. Then low ionisation states, such as OI and CII, may be more common.

Furlanetto \& Loeb (2003) constructed a reasonably simple model to examine a range of possible enrichment histories and their observable implications. The model uses a Monte Carlo implementation of the extended Press-Schechter formalism (Lacey \& Cole 


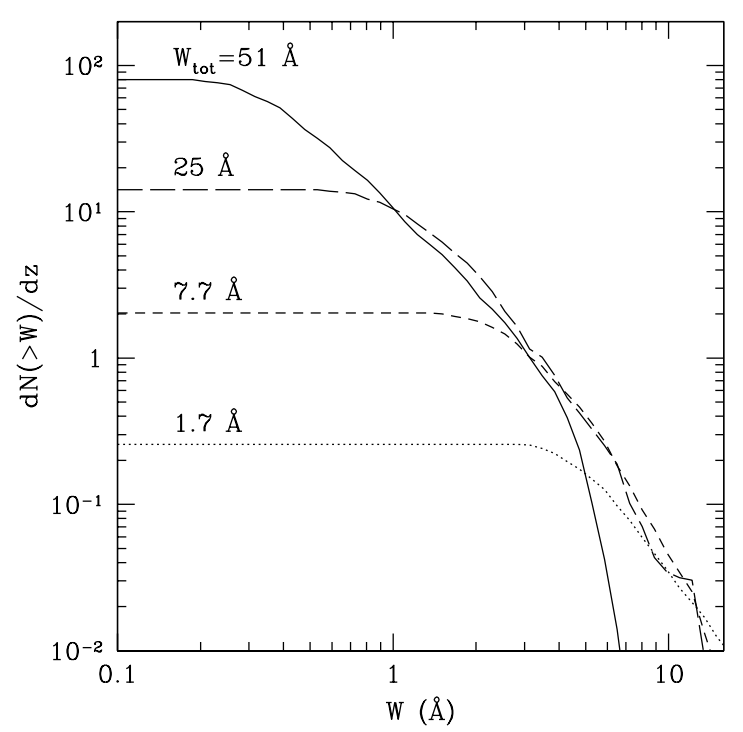

Figure 4. Number of OI $\lambda 1302$ systems intersected along a random line of sight per unit redshift, as a function of (observed) equivalent width $W$. From top to bottom, the curves are for $z=8,12,16$, and 20. Each curve is also marked with the total integrated absorption per unit redshift. See Furlanetto \& Loeb (2003) for details.

1993) to describe the star formation history of galaxies and assumes a spherical, thinshell, pressure-driven outflow to follow the wind expansion. The most important input parameters are the star formation efficiency (which we take to be $f_{\star}=0.1$ here), the fraction of supernova energy available to the wind $\left(f_{w}=0.25\right)$, and the stellar IMF (which determines the supernova frequency, the metal yield, and the supernova energy; we use Pop II values here). Fig. 3 shows the resulting wind sizes. As expected, small galaxies are by far the most efficient polluters: this is because gravity confines winds around massive halos. Given the steep mass function at high redshifts, dwarf galaxies thus dominate the enrichment.

Armed with the wind sizes and their metal content, it is easy to compute the absorption statistics. We assume that the winds remain spherical, do not fragment, and contain all of the metals produced by the host galaxy (obviously an upper limit). We also neglect clustering of the hosts. Fig. 4 shows the resulting absorption at a range of redshifts. Note that these curves correspond to a filling factor of enriched material $Q \sim 10^{-4}, 10^{-3}, 10^{-2}$, and 0.1 , from bottom to top. Obviously, the absorption is never tremendously strong, but it is substantial even when $Q \sim 1-10 \%$. For example, we expect $\sim 10$ absorbers per unit redshift with equivalent width $W>1 \AA$ once $Q>1 \%$. We have chosen OI $\lambda 1302$ for this figure; however, a number of other transitions, for both low- and high-ionisation states, yield comparable amounts of absorption.

The model suggests a number of observable trends. First, the total absorption depends on the total mass of metals ejected into the IGM, but the distribution of absorber strengths depends on the source parameters. Powerful winds from small halos produce widely distributed weak absorption, while less efficient winds from massive galaxies create only a few features (albeit strong ones). Thus we can directly study the metal ejection process. This is more difficult at lower redshifts because by that time the winds have merged, fragmented, etc. Second, the strongest absorbers appear at high redshift, 
because the winds are most compact then. Third, comparing the strengths of different metal lines constrains the metal yield, allowing us to distinguish enrichment by Pop II and Pop III stars. Finally, extrapolating Fig. 4 to filling factors near unity, metal absorption offers a powerful probe of the most extreme enrichment scenarios, in which an early generation of Pop III stars pollutes the entire Universe, even if only to a low level.

\subsection{The "OI forest"}

Oh (2002) pointed out another extremely interesting possibility. OI has an ionisation potential of $13.62 \mathrm{eV}$; as a result it remains in tight charge-exchange equilibrium with $\mathrm{HI}$ in most astrophysical environments. Therefore we will see OI $\lambda 1302$ absorption wherever the Universe is neutral (and enriched). For a fully neutral medium at the mean density, the optical depth will be near unity for $Z \approx 10^{-1.5} \mathrm{Z}_{\odot}$; thus, we might expect to see an "OI forest" marking regions of dense neutral material. Unlike the Ly $\alpha$ forest, these features will not be saturated, making them useful measures when $x_{\mathrm{HI}} \approx 1$ (although there is a degeneracy with the metallicity, of course). Oh (2002) showed that we would expect observable features to occur wherever the line-of-sight passes through an optically thick Lyman-limit cloud, which are obviously common before reionisation.

Of course, such clouds must be enriched to a reasonable level. Oh (2002) argued that this is likely to be the case, because such regions typically lie in dense environments relatively near to galaxies and hence trace the same underlying density field. They are therefore probably among the first pockets in the IGM to be polluted by metal-bearing winds. On the other hand, they will be among the last to become highly ionised, because their recombination time is short (Miralda-Escudé et al. 2000). The OI forest should therefore appear even if enrichment is not yet complete, and it offers a powerful probe of the tail end of reionisation.

\section{Discussion}

In the past several years, quasars have finally taken us to the reionisation era - or at least its final stages. The SDSS objects have convincingly demonstrated that the IGM is evolving dramatically at $z \approx 6$, with a rapid increase in the optical depth along all lines of sight. However, the two well-studied spectra show remarkable variations on large $(\sim 100$ co-moving Mpc) scales, with one remaining highly ionised and one showing complete saturation. This large-scale behaviour is surprising given that structure formation remains in its early stages, but recent models of reionisation have shown that inhomogeneity is actually an integral part of reionisation and can be traced to the strong clustering of high-redshift galaxies. These models predict a topology of large ionised bubbles floating in an ocean of neutral gas; the typical bubbles stretch across tens of co-moving Mpc when $x_{\mathrm{HI}}>0.75$. Simple models suggest that fluctuations similar to those observed should appear even in the middle stages of reionisation! They will allow us to study the transition between the "bubble-dominated" radiation field characteristic of reionisation and the "web-dominated" version characteristic of the later Universe.

We would also like to use quasars to study the physical processes driving the ionising sources; one key question is the role of metal enrichment (as well as its importance for the lower redshift IGM; see A. Aguirre in this volume). We have argued that this process can be directly observed even after Ly $\alpha$ absorption saturates, because many metals have strong transitions red-ward of Ly $\alpha$. If a substantial fraction $(>1 \%)$ of the Universe is enriched, significant metal absorption should appear along most lines of sight. Such observations offer an indirect route to study the physics of winds and the properties of their hosts. Moreover, because OI sits in tight charge-exchange equilibrium with HI, it 
also traces neutral gas. Oh (2002) showed that the resulting OI forest is a useful diagnostic of the tail end of reionisation.

Of course, exploring either of these avenues is technically challenging. Even in the bestcase scenarios, Lyman-series transmission from $z>6$ is weak and fairly rare, requiring large samples of quasars (or gamma-ray bursts) for good statistics. Metal lines fall in a difficult spectral region at $z>6$, where the night-sky features are complex and variable. Nevertheless, both techniques offer powerful probes of the high-redshift Universe and will open new windows into the reionisation process and the physics of the ionising sources. Solving these challenges should be a high priority in the coming years.

\section{Acknowledgements}

I would like to thank my collaborators on the projects described here for sharing their ideas and work: L. Hernquist, A. Loeb, S. P. Oh, and M. Zaldarriaga. I would also like to thank the conference organisers for arranging a fascinating meeting.

\section{References}

Abel, T., Bryan, G. L., Norman, M. L., 2002, Science, 295, 93

Barkana, R., 2002, New Astron., 7, 85

Barkana, R., Loeb, A., 2001, Phys. Rep., 349, 125

Barkana, R., Loeb, A., 2004, ApJ, 609, 474

Bromm, V., Coppi., P. S., Larson, R. B., 2002, ApJ, 564, 23

Cen, R., 2003, ApJ, 591, L5

Ciardi, B., Stoehr, F., White, S. D. M., 2003, MNRAS, 343, 1101

Dekel, A., Silk, J., 1986, ApJ, 303, 39

Fan, X., et al., 2001, AJ, 122, 2833

Fan, X., et al., 2002, AJ, 123, 1247

Furlanetto, S. R., Hernquist, L., Zaldarriaga, M., 2004b, MNRAS, 354, 695

Furlanetto, S. R., Loeb, A., 2003, ApJ, 588, 18

Furlanetto, S. R., Loeb, A., 2005, ApJ, in press, astro-ph/0409656

Furlanetto, S. R., Oh, S. P., 2005, in preparation

Furlanetto, S. R., Zaldarriaga, M., Hernquist, L., 2004a, ApJ, 613, 1 [FZH04]

Gunn, J. E., Peterson, B. A., 1965, ApJ, 142, 1633

Kogut, A., et al., 2003, ApJS, 148, 161

Lacey, C., Cole, S., 1993, MNRAS, 262, 627

Madau, P., Ferrara, A., Rees, M. J., 2000, ApJ, 555, 92

Mesinger, A., Haiman, Z., 2004, ApJ, 611, L69

Miralda-Escudé, J., Haehnelt, M., Rees, M. J., 2000, ApJ, 530, 1

Oh, S. P., 2002, MNRAS, 336, 1021

Oh, S. P., Furlanetto, S. R., ApJ, 620, L9

Scannapieco, E., Ferrara, A., Madau, P., 2002, ApJ, 574, 590

Shapiro, P. R., Iliev, I. T., Raga, A. C., 2004, MNRAS, 348, 753

Sokasian, A., Abel, T., Hernquist, L., Springel, V., 2003, MNRAS, 344, 607

Sokasian, A., Yoshida, N., Abel, T., Hernquist, L., Springel, V., 2004, MNRAS, 350, 47

White, R. L., Becker, R. H., Fan, X., Strauss, M. A., 2003, AJ, 126, 1

White, R. L., Becker, R. H., Fan, X., Strauss, M. A., 2005, submitted to AJ, astro-ph/0411195

Wyithe, J. S. B., Loeb, A., 2003, ApJ, 588, L69

Wyithe, J. S. B., Loeb, A., 2004, Nature, 427, 815 